\title{
HEV and HBV Dual Infection: A Review
}

\author{
Myra Nasir* and George Y. Wu \\ Department of Medicine, Division of Gastroenterology-Hepatology, University of Connecticut Health Center, Farmington, CT, USA
}

\begin{abstract}
Hepatitis E virus (HEV) is a global health problem, affecting about 20 million people worldwide. There is significant overlap of hepatitis B virus (HBV) and HEV endemicity in many Asian countries where dual infections with HEV and HBV can occur. Though the clinical course of HEV is largely self-limited, HEV superinfection in patients with chronic hepatitis B (CHB) can result in acute exacerbation of underlying $\mathrm{CHB}$. HEV superinfection in patients with CHB-related cirrhosis has been identified as a risk factor for decompensated cirrhosis and an independent predictor of mortality. Whereas acute HEV infection in pregnancy can cause fulminant liver failure, the few studies on pregnant patients with dual HBV and HEV infection have shown a subclinical course. Immunosuppression is a risk factor for the development of chronic HEV infection, which can be managed by decreasing the dose of immune-suppressants and administering ribavirin. Vaccination for HEV has been developed and is in use in China but its efficacy in patients with $\mathrm{CHB}$ has yet to be established in the USA. In this review, we appraise studies on dual infection with HEV and HBV, including the effect of HEV superinfection and coinfection in $\mathrm{CHB}$, management strategies used and the role of active vaccination in the prevention of HEV.
\end{abstract}

Citation of this article: Nasir M, Wu GY. HEV and HBV dual infection: A review. J Clin Transl Hepatol 2020;8(3):313-321. doi: $10.14218 /$ JCTH.2020.00030.

\section{Introduction}

Hepatitis E virus (HEV) infection is a global health problem, affecting about 20 million people yearly and is responsible for 44,000 deaths reported in 2015 worldwide. $^{1}$ Though its

Keywords: Hepatitis B; Hepatitis E; Coinfection; Superinfection; Chronic hepatitis B.

Abbreviations: $A C L F$, acute-on-chronic liver failure; ALT, alanine transaminase; AST, aspartate aminotransferase; CHB, chronic hepatitis $B$; CLD, chronic liver disease; CTP, Child-Turcotte-Pugh; CLIF-C ACLF, Chronic Liver Failure-Consortium Acute-on-Chronic Liver Failure; CLIF-C OFs, Chronic Liver Failure-Consortium Organ Failure; COSSH-ACLFs, Chinese Group on the Study of Severe Hepatitis B - Acute-on-Chronic Liver Failure; DNA, deoxyribonucleic acid; ELISA, enzymelinked immunosorbent assay; GGT, gamma-glutamyl transpeptidase; HAV, hepatitis $A$ virus; $\mathrm{HBeAg}$, hepatitis $B$ e antigen; $\mathrm{HBsAg}$, hepatitis $B$ surface antigen; $\mathrm{HBV}$, hepatitis B virus; HCV, hepatitis C virus; HDV, hepatitis D virus; HEV, hepatitis E virus; HIV, human immune deficiency virus; INR, international normalized ratio; LFT, liver function test; MELD, Model for End-Stage Liver Disease; OR, odds ratio; RNA, ribonucleotide acid; SOFA, Sequential Organ Failure Assessment; TGF $\beta$, tissue growth factor $\beta$.

Received: 10 April 2020; Revised: 29 May 2020; Accepted: 7 June 2020

*Correspondence to: Myra Nasir, Department of Medicine, Division of Gastroenterology-Hepatology, University of Connecticut Health Center, 263 Farmington Avenue, Farmington, CT 06030, USA. Tel: +1-860-470-6616, E-mail: mnasir@uchc.edu clinical course is largely acute and self-limited, HEV infection can run a fulminant course in pregnant women, especially in the third trimester, with case-fatality ratios ranging from $10 \%$ to $42 \% .^{2}$ Immunocompromised patients, such as those with human immune deficiency virus (HIV) infection, transplant recipients on immunosuppressants and those receiving chemotherapy, can have persistent carriage of HEV virus with chronic infection. ${ }^{3,4}$

Chronic hepatitis $B$ virus (CHB) infection is defined by hepatitis $B$ surface antigen ( $\mathrm{HBsAg}$ ) positivity for greater than 6 months. Because hepatitis B virus (HBV) and HEV are highly prevalent in many areas of the world, the likelihood of dual infections in these areas is also high. Although HEV monoinfection tends to be mild, superinfection or coinfection with other viruses can present additional risks. In such dual infections, HEV can superinfect (defined as anti-HEV antibody and/or HEV RNA seroconversion in patients with CHB who were initially negative for these antibodies or HEV RNA). Alternatively, HEV and HBV can coinfect a person negative for both HBsAg and HEV antibodies prior to infection. Though HEV spread mainly occurs through the fecal-oral route, it can also be transmitted by blood transfusion in areas endemic to $\mathrm{HEV}^{5,6}$ Since these areas are also endemic to HBV, this can lead to HEV-HBV coinfection.

Superinfection with HEV has been reported to cause acute exacerbations of asymptomatic $\mathrm{CHB}$ infection, which may result in severe complications and poor outcomes. ${ }^{4,7}$ This is supported by results from a cell culture transcriptome-based analysis, which showed enhanced expression of proinflammatory genes in HEV-only and HEV+HBV-infected cell groups as compared to HBV-only-infected cells, whereas hepatocyte destruction occurred in all three groups of cells. ${ }^{8}$

This review will discuss the viral interaction between HEV and HBV, as well as the related effects on severity of liver disease in patients with underlying $\mathrm{CHB}$ and possible management strategies.

\section{Epidemiology}

HEV is hyperendemic in many Asian countries, such as India, Bangladesh, Pakistan and China, where genotypes 1 and 2 are common and are transmitted mainly through the fecaloral route. Developed countries, such those in North America and Europe, have reported increasing cases of genotype 3 and 4, which mainly involve zoonotic transmission routes, including eating of raw pig or deer meat. ${ }^{9}$

HBV prevalence (determined by HBsAg positivity) is high $(\geq 8 \%)$ in many African countries and Central America, intermediate $(2 \%$ to $7 \%)$ in India, Pakistan, China and Canada, but low $(<2 \%)$ in Western Europe and the USA. ${ }^{10}$ There is significant overlap of HBV and HEV endemicity in 
China, India, Pakistan and Bangladesh, where dual infections with HEV and HBV can occur.

Studies of serum epidemiology conducted in China showed superinfection/coinfection with two or more hepatitis viruses in about $40 \%$ of the patients, where HEV superinfection in patients with $\mathrm{CHB}$ account for $17.6 \%{ }^{11}$ Another study reported HEV superinfection rate in $\mathrm{CHB}$ patients to be $13.7 \%$ (compared to $54 \%$ in patients with hepatitis C). ${ }^{12} \mathrm{~A}$ study conducted in India found $2.8 \%(26 / 927)$ of patients to have both HBsAg and anti-HEV IgM positivity, reflecting prevalence of hepatitis $E$ and hepatitis $B$ dual infection. ${ }^{13}$

However, all the above studies used anti-HEV antibodies to demonstrate prevalence, which may have yielded underestimations due to false negatives, especially in immunocompromised CHB patients.

Certain populations might be at higher risk of developing dual infection with HEV and HBV. In Western countries, more than $60 \%$ of organ transplant recipients infected with HEV develop chronic hepatitis leading to cirrhosis. ${ }^{14,15}$ These patients include, among others, liver transplant recipients with $\mathrm{CHB}$, which itself can give rise to dual HEV-HBV infections. There is a high prevalence of HIV-HBV coinfection, with serological markers of past or current HBV infection present in one-third of the HIV-infected patients. ${ }^{16}$ HIV-infected patients have a higher rate of exposure to HEV than the general population and chronic hepatitis $E$ infection has been described in HIV-infected patients, making them susceptible to HEV-HBV dual infection. ${ }^{3,17}$ Similarly, incarcerated individuals are at high risk for acquiring HEV, mainly owing to low socioeconomic backgrounds, and other blood-borne diseases, such as HBV and HIV, due to high risk behaviors, including intravenous drug use (which is more common in the incarcerated population as compared to the general population). ${ }^{18,19}$ Higher HEV prevalence has also been noted in dialysis patients in HEV endemic areas, which increases the prevalence of dual HEV-HBV infections. ${ }^{20}$

However, large, population-based studies are required to accurately assess the prevalence of HBV-HEV dual infections in the above-mentioned populations, as compared with the general population.

\section{Effects of dual infection on severity of liver disease}

\section{Superinfection in patients with cirrhosis}

Studies have shown that HEV superinfection in patients with cirrhosis results in higher mortality with rapid hepatic decompensation (Table 1). Acharya et al. ${ }^{21}$ recruited 107 patients with cirrhosis, of which 30 (28\%) had detectable HEV RNA. All patients with cirrhosis were divided into three groups, with Group I consisting of patients with rapid decompensation, Group II with chronically decompensating cirrhosis, and Group III with stable cirrhosis. Group I included 42 patients, out of which 18 had HBV and 7 had HBV+hepatitis C virus (HCV) infection. Twenty one out of all thirty patients with HEV RNA positivity (70\%) belonged to Group I due to their rapid decompensation status. Within the Group I, patients with HEV infection, when compared with those without the infection, had significantly higher mortality at 4 weeks ( $43 \%$ vs. $22 \%, p=0.001)$ and at 12 months (70\% vs. $30 \%$, $p=0.001$ ). Multivariate analysis demonstrated HEV RNA positivity to be an independent predictor of mortality (relative risk 1.88, 95\% confidence interval: 1.01-3.49) in these patients. Use of HEV RNA to demonstrate HEV positivity and multivariate analysis, as mentioned, increased the reliability of the study findings. Although healthy controls were included in this study, lack of controls including non-viral cirrhotic patients with superinfection with HEV is a limitation.

Hamid et al. ${ }^{22}$ documented four patients with CLD who were found to have acute HEV infection (HEV IgM-positive serologies) with subsequent decompensation, including worsening ascites or development of encephalopathy. One patient out of the four had CHB. Kumar et al. ${ }^{23}$ studied 32 patients with decompensated liver cirrhosis. Out of the 32 patients, 14 (44\%) were found to have anti-HEV IgM as compared to only 3 out of 48 patients (6\%) with stable cirrhosis $(p<0.0001)$. Of the 14 patients with HEV infection in the setting of decompensated cirrhosis, 8 had CHB-related cirrhosis.

The above studies have several limitations. These were retrospective analyses and included only a small number of patients, using commercial enzyme-linked immunosorbent assay (ELISA) tests to detect anti-HEV IgM to demonstrate acute HEV infection. These tests unfortunately have low sensitivity in endemic areas, which is where these studies were conducted. Additionally, patients with cirrhosis may have impaired cell-mediated and humoral immunity and so the presence of anti-HEV IgM may not be a reliable marker for active infection in this patient population. ${ }^{24,25}$ HEV RNA may have been a more reliable marker of infection in these patients. Although the increased risk of poor prognosis in cirrhotics who become superinfected with HEV seems well supported, that is not surprising since the same risks have been shown with other viral superinfections, such as with hepatitis A virus (HAV) and hepatitis D virus (HDV). ${ }^{26,27}$ As such, these cases do not shed light on the risk of poor prognosis in $\mathrm{CHB}$ cases without cirrhosis that are dually infected with HEV.

Chen et al. ${ }^{28}$ recruited 228 patients with acute HEV infection (anti-HEV IgM-positive) and CHB (HBsAg positivity and/ or HBV DNA positivity for over 6 months). Patients with and without cirrhosis who contracted HEV superinfection had higher rates of complications of liver disease in the patients with cirrhosis $(77.7 \%$ vs. $28.4 \%, p<0.001)$ and a higher short-term mortality rate compared to patients without cirrhosis $(20 \%$ vs. $7.5 \%, p=0.002)$. Complications of liver disease in this study included ascites, hepatic encephalopathy, gastrointestinal hemorrhage, or bacterial infections. Mortality was defined as death due to liver disease within 3 months of disease onset. When patients with compensated cirrhosis were compared with patients with decompensated cirrhosis, no significant difference was found in the shortterm mortality and laboratory tests (alanine transaminase (ALT), aspartate aminotransferase (AST), total bilirubin, albumin, international normalized ratio (INR), and platelet count). Regarding disease severity in coinfected patients with cirrhosis, there was a higher percentage of patients in the severe disease group who were smokers, as compared to the patients in the mild disease group (37\% vs. $14.3 \%$, $p<0.05$ ). No significant difference in disease severity was noted when other factors (hypertension, diabetes, kidney disease, other liver diseases, or potential hepatotoxic medication use) were evaluated. In noncirrhotic patients, alcohol use (odds ratio (OR): 6.4, $p=0.020$ ), diabetes (OR: 7.5, $p=0.003$ ), and kidney diseases (OR: 12.7, $p=0.005$ ) were noted as independent predictors for severe disease by multivariate analysis.

Though this is a retrospective study, 228 patients with HEV-HBV dual infection were recruited, which is a much larger 


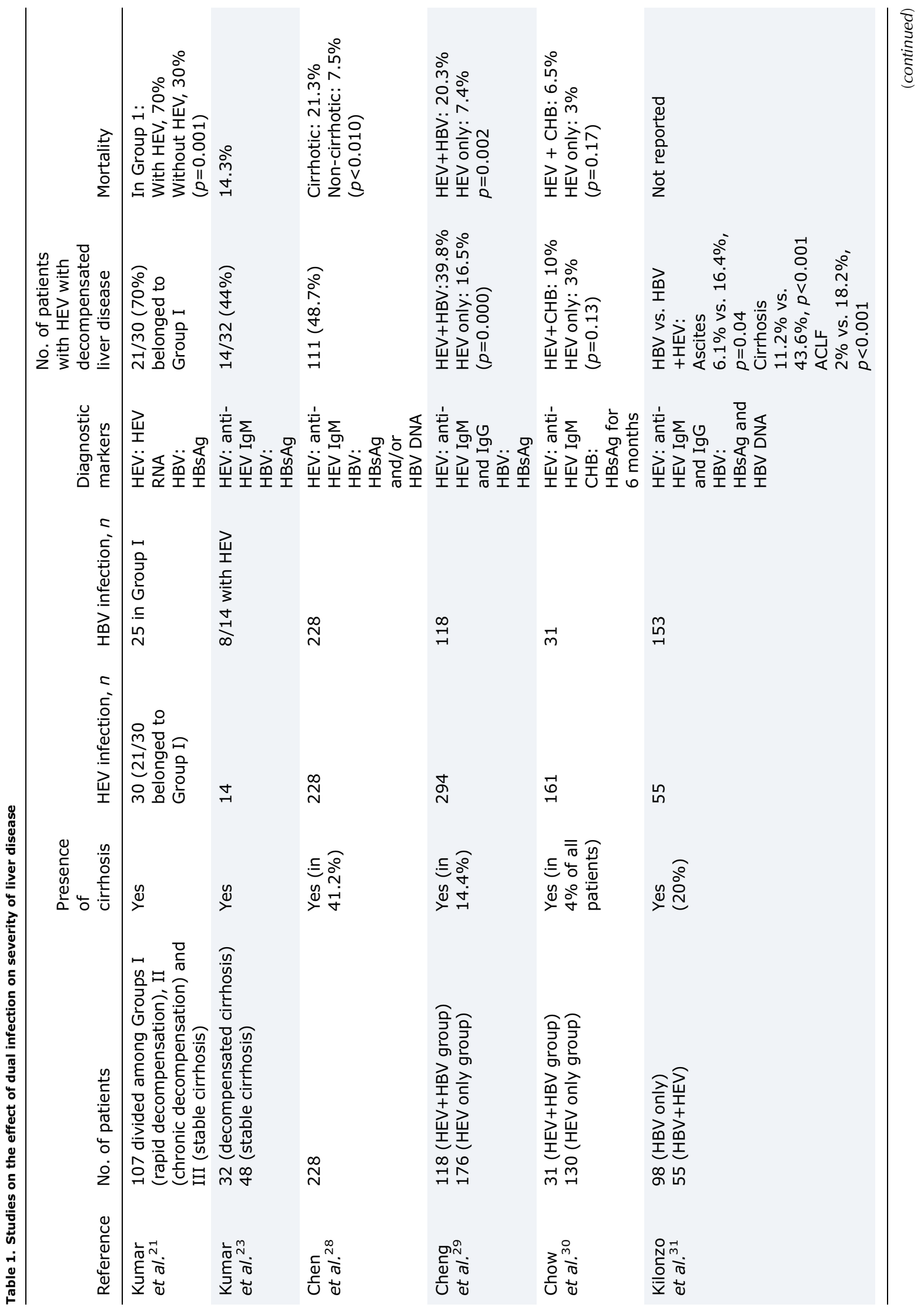




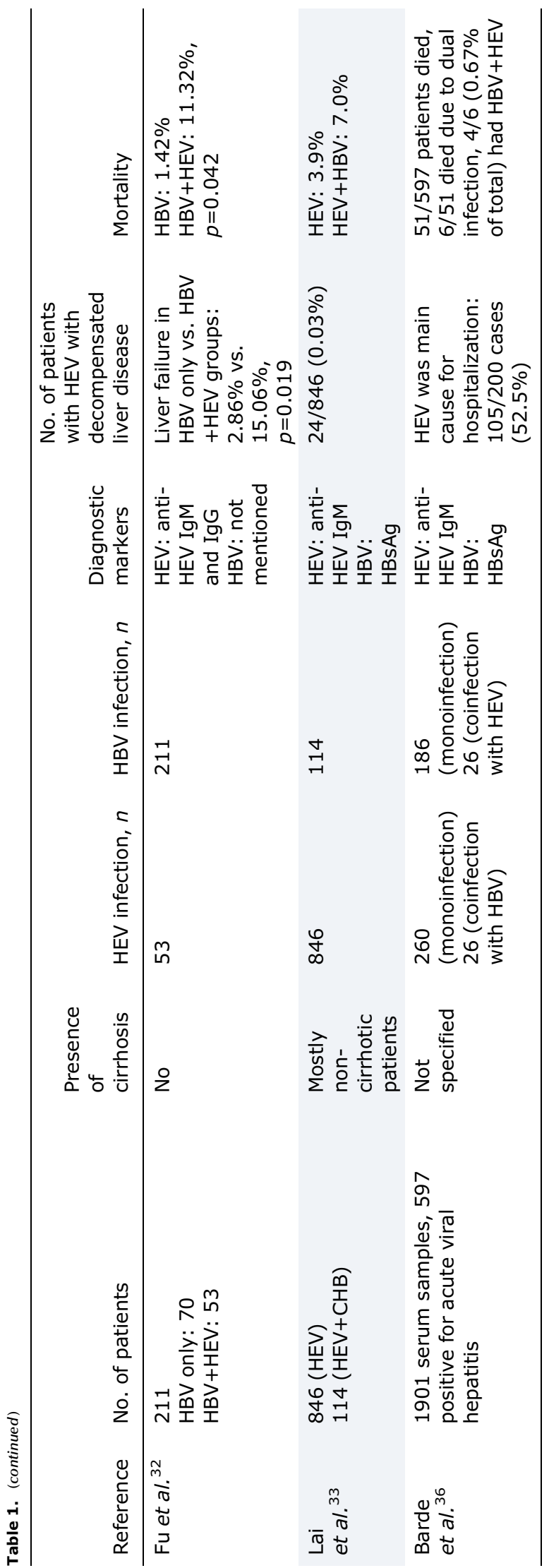

dual-infected population than that in the other mentioned studies. Additionally, use of multivariate analysis adjusts for confounding factors, allowing more reliable findings. However, this study used anti-HEV antibodies as diagnostic markers instead of the more sensitive HEV RNA test, which may have resulted in false negatives with underestimation of the number of patients with HEV.

To evaluate the influence of CHB on acute HEV infection, Cheng et al. ${ }^{29}$ studied 118 patients with HBV and HEV, out of which $16.9 \%$ were hepatitis B e antigen (HBeAg)-positive, $55.1 \%$ were HBV DNA-positive, and $14.4 \%$ had underlying cirrhosis. A control group included 176 patients with acute HEV infection alone. When the two groups were compared, the dual infected group was noted to have a higher mortality, with more patients developing liver failure compared to the HEV monoinfected group $(p=0.002$ and $p=0.000$, respectively). Among the 118 patients with HBV and HEV, the incidence of liver failure and death compared between HBeAgpositive and -negative groups and between HBV DNA-positive and -negative groups showed no significant difference. However, Model for End-Stage Liver Disease (MELD) scores, occurrence of liver failure and complications of liver disease (ascites, peritonitis, hepatic encephalopathy, gastrointestinal bleeding, and hepatorenal syndrome) were significantly higher for the cirrhotic patients as compared to the non-cirrhotic patients ( $p=0.03, p=0.01$ and $p=0.00$, respectively). Major limitations of this study include its retrospective design and possible underestimation of HEV infection (since HEV RNA was not consistently checked). Additionally, it is unclear if the control group consisting of patients with acute HEV infection included cirrhotics or not.

Chow et al., ${ }^{30}$ in a 10-year retrospective study, compared outcomes in patients with acute HEV and $\mathrm{CHB}$ with those with HEV only. Cirrhosis was found in $2 \%$ of the HEV-only group compared with $13 \%$ of the HEV and CHB group $(p=0.03)$. The latter group was found to have higher liver failure rate, liverrelated mortality, and all-cause mortality. However, these findings were not statistically significant, likely due to the small patient population included in the study.

Nevertheless, the overall evidence demonstrated by the studies mentioned above suggests that HEV superinfection increased the risk of hepatic decompensation and increased mortality in $\mathrm{CHB}$ patients with cirrhosis.

\section{Superinfection in patients without cirrhosis}

One study included 153 patients who were divided in two groups ( those being the HBV monoinfection (98/153) and HBV-HEV superinfection (55/153) groups. ${ }^{31}$ Of the total 153, about $80 \%$ were non-cirrhotics. HBV infection was determined using HBsAg and HBV DNA positivity, and HEV infection was determined using anti-HEV IgG and IgM antibodies. None of the patients were on anti-viral therapy. Baseline clinical findings were compared between the two groups, which was remarkable for significantly higher prevalence of acute-onchronic liver failure (ACLF) $(p<0.001)$ and ascites, hepatorenal syndrome, and encephalopathy $(p=0.04)$ in patients with HEV superinfection. Comparison of laboratory findings between the two groups showed significantly higher liver enzymes (ALT, AST, gamma-glutamyl transpeptidase (GGT) and bilirubin) with lower albumin levels and HBV viral load in patients with HBV-HEV superinfection $(p<0.001)$. These are baseline findings. Since this is a retrospective study, the authors only speculated that the decompensation developed 
after HEV infection. It is important to note that the decompensation (ascites, hepatorenal syndrome, encephalopathy, etc.) was present at baseline when the patients were enrolled in the study, and it is unclear if the complications of liver disease in patients made them susceptible to HEV superinfection or the latter caused a progression in the liver disease.

Fu et al. ${ }^{32}$ studied clinical and prognostic effects of superinfection with other hepatitis viruses on patients with CHB. The study population consisted of 211 patients, out of which 53 had both HBV and HEV. Patients with $\mathrm{CHB}+\mathrm{HEV}$ were noted to have a higher percentage of individuals with almost all clinical effects studied, such as fever, decreased appetite, nausea and vomiting, epigastric discomfort and hepatosplenomegaly, compared to those patients with $\mathrm{CHB}$ alone. However, more data regarding whether these differences were statistically significant or not were not provided. Laboratory findings such as mean ALT, AST and total bilirubin were higher $(p<0.05)$ and clinical outcomes such as liver failure and death had a higher incidence in patients with $\mathrm{CHB}$ and HEV compared to patients with $\mathrm{CHB}$ alone $(p<0.05)$. Though this was a retrospective, single-center study with a small study population, its overall findings correspond with the findings of other studies mentioned above. Additionally, unlike the previously mentioned studies, this one was specific to effects of HEV superinfection in patients with $\mathrm{CHB}$.

Lai et al. ${ }^{33}$ studied 1914 patients with either HEV or HAV infection in a retrospective study aimed at assessing the clinical outcomes of the patients with and without CHB. Positive $\mathrm{HBsAg}$ status was found to be the independent predictor of 30-day liver-related mortality in HEV-infected patients, with an adjusted hazards ratio of $3.344(p=0.020)$. Significantly more patients with acute HEV had underlying $\mathrm{CHB}$, as compared to HAV-infected patients $(13.5 \%$ vs. $8.0 \%, p=0.001)$. Though this study includes a large sample size, the data were collected from a local registry, using ICD-9 codes to retrieve data. This, thus, involves the limitation of possible coding errors by physicians and missing data from the cohort, which can result in inaccurate results.

Another study evaluated prognostic models to determine which patients with CHB with HEV superinfection would develop ACLF and have poor prognosis. ${ }^{34}$ Of the 193 patients with $\mathrm{CHB}$ who were admitted to the hospital with acute HEV infection (positive anti-HEV IgM), 13 were diagnosed with ACLF on admission and 13 progressed to ACLF. Five prognostic models (Child-Turcotte-Pugh (CTP) score, MELD score, Chronic Liver Failure-Consortium Acute-on-Chronic Liver Failure (CLIF-C ACLFs), Chronic Liver Failure-Consortium Organ Failure (CLIF-C OFs) and Chinese Group on the Study of Severe Hepatitis B - Acute-on-Chronic Liver Failure (COSSH-ACLFs)) were used to predict outcomes of these ACLF patients. In predicting the development of ACLF, CTP score had higher performance compared with MELD score $(p<0.05)$ and COSSH-ACLFs had the highest performance in predicting poor outcomes among the ACLF patients when compared with the other four models $(p<0.05)$. The CTP score includes total bilirubin, albumin, ascites, hepatic encephalopathy and prothrombin time, whereas COSSH-ACLFs includes INR, HBV Sequential Organ Failure Assessment (SOFA), age and total bilirubin. However, it is important to note that this was a retrospective, single-center study and larger, multicenter studies are required to validate these findings. Despite the limitations of the studies mentioned above, they all consistently demonstrated decompensation of liver disease in CHB patients with HEV superinfection.

\section{Coinfection}

Bouamra et al. ${ }^{35}$ reported on the case of a 41 year-old male with history of HIV, CD4 count of $222 / \mathrm{mm}^{3}$ and undetectable HIV RNA levels, who presented with abdominal pain and dark urine, and was found to have elevated liver function test (LFT) results in September 2010. He was noted to be negative for HBsAg in December 2009 but retesting in July 2010 revealed HBsAg positivity. HBV DNA was undetectable at that time. At his presentation in September 2010, he was found to have HBV DNA levels $>9 \log _{10} \mathrm{IU} / \mathrm{mL}$ and anti-HB core IgM positivity. Anti-HEV IgM and HEV RNA were also detectable. He was treated with ribavirin for HEV with undetectable HEV RNA levels within 2 weeks and liver biomarkers returned to normal within 4 months. He received tenofovir and emtricitabine for HBV treatment and had undetectable viral count at 14 months after treatment. This report demonstrates the disease course of a patient who developed acute HBV and HEV coinfection and was successfully treated with antivirals. It is rare to observe coinfections with viruses that are transmitted through different routes, such as in this case. Though this case sheds light on the importance of testing for both HEV and HBV in patients with symptoms of acute hepatitis, casecontrolled studies are required to elicit the effects of coinfection on disease severity and mortality.

Barde et al. ${ }^{36}$ tested 1901 serum samples of patients admitted with acute viral hepatitis for HEV (anti-HEV IgM), HBV (HBsAg), HAV (anti-HAV IgM) and HCV (anti-HCV antibodies). Coinfection with HBV and HEV was found in 25 samples. Out of the six patients who died due to dual infection, four had HEV-HBV co-infection and two had HEV-HCV co-infection. Case fatality was higher in patients with acute viral hepatitis due to coinfection, as compared to monoinfection cases ( $14.3 \%$ vs. $8.1 \%)$. However, a major limitation in the study was the lack of testing samples for other serologic markers for HBV, such as hepatitis B core antibody, IgM and IgG, which makes it unclear if the infection is acute or chronic.

\section{Effect of dual infection on viral replication}

There are scarce and conflicting data regarding the replication of viruses in dual infected patients. A study recruited 43 patients positive for HBsAg who were divided into Group 1 ( 21 patients positive for $\mathrm{HBeAg}$ ) and Group 2 (22 patients negative for $\mathrm{HBeAg}$ ) at presentation. ${ }^{7}$ Group 1 had no patients with acute HEV infection (i.e. anti-HEV IgM-positive), whereas in Group 2, 27.3\% had acute HEV and $13.6 \%$ had both acute HEV and HAV (anti-HAV IgM) infections. The median HBV DNA levels in patients with exacerbation of liver disease in Group 2 (defined as ALT >400 U/L) were lower in patients with HEV (3.679 log copies/mL) and HEVHAV ( $\leq 3.70$ log copies $/ \mathrm{mL}$ ) superinfections than in those with HBV alone (5.36 log copies/mL). However, it is unclear if this difference was statistically significant or not. Additionally, baseline HBV DNA levels are not available to compare with HBV DNA levels after HEV superinfection. Zhang et al. ${ }^{37}$ in a similar study demonstrated no significant difference in the HBV DNA levels between a group of patients with $\mathrm{CHB}+\mathrm{HAV}$ superinfection and $\mathrm{CHB}+\mathrm{HEV}$ superinfection $(p=0.176)$. However, there was no comparison with acute exacerbation of liver disease in patients with $\mathrm{CHB}$ only and baseline HBV DNA levels are not available to compare with HBV DNA levels after HEV superinfection. 
Yeh et al. ${ }^{38}$ reported a case of seroclearance of HBsAg in a renal transplant patient with dual chronic HBV and HEV infection after an acute-on-chronic HEV infection. However, it is impossible to draw this conclusion with a single case and further studies are required to evaluate this hypothesis.

Kilonzo et al. ${ }^{31}$ found patients with HBV monoinfection to have a higher peak of HBV viral load as compared with those with HEV superinfection $(p<0.001)$. A proposed explanation was that since HEV is an RNA virus, it may function as a ribozyme in the inhibition of HBV DNA replication. However, the retrospective design of the study with a small study sample were major limitations. Larger prospective studies are required to further evaluate the effect of HEV superinfection on HBV viral load.

Hoan et al. ${ }^{39}$ recruited 1318 patients with HBV and 340 healthy controls, and found higher levels of HBV DNA (median of $>10^{6}$ copies per $\mathrm{mL}$ ) in patients with acute HEV infection, as compared with those with past infection (median of $\sim 10^{5}$ copies per $\mathrm{mL}$ ) and those with no infection (median of $<10^{6}$ copies per $\left.\mathrm{mL}\right)(p=0.026)$. Though this study was conducted on a large scale, it primarily used ELISA for antibody testing, which, due to its low sensitivity, may have underestimated the number of patients with HEV superinfection.

Schulz et al. ${ }^{40}$ reported on the case of a patient with $\mathrm{CHB}$ with undetectable HBV DNA levels (or $<250 \mathrm{IU} / \mathrm{mL}$ ). At a 3mo follow-up (interval), he was noted to have deranged LFT results (ALT of $1010 \mathrm{U} / \mathrm{L}$, AST pf $338 \mathrm{U} / \mathrm{L}$, GGT of $307 \mathrm{U} / \mathrm{L}$, with normal bilirubin) with low HBV viral load (145 IU/mL) with HEV RNA levels of 76,400 copies/mL. Over a month later, the patient developed fatigue and generalized weakness, at which time his HBV viral load was noted to be $23,200 \mathrm{IU} / \mathrm{mL}$, with positivity for anti-HEV IgG, unclear anti-HEV IgM results and undetectable HEV RNA levels. HAV and HCV infections were ruled out. In subsequent follow-ups, the patient's liver enzymes declined and HBV DNA levels declined back to $<141$ $\mathrm{IU} / \mathrm{mL}$. At 5 months after his initial work up, the patient had undetectable HBV DNA and HEV RNA levels. This case shows the occurrence of a transient increase in HBV DNA levels after acute HEV infection, with eventual decline after HEV RNA level decline. Prospective studies are required to validate this finding, however.

Shang et al. ${ }^{41}$ conducted a study on the effect of HEV superinfection on HBV viral replication in patients with $\mathrm{CHB}$ by measuring the HBV DNA levels in serum and liver tissue. It was observed that the $\mathrm{CHB}$ patients with HEV superinfection had lower levels of HBV DNA in serum and liver tissue (5.45 and 6.96 copies $/ \mathrm{mL}$, respectively), as compared with $\mathrm{CHB}$ patients without HEV superinfection (6.59 and 8.47 copies/ $\mathrm{mL}$, respectively, $p<0.05)$. HBeAg and hepatitis B core antigen positivity rates were also noted to be significantly lower in the CHB patients with HEV superinfection.

Though there are more studies demonstrating lower HBV replication in $\mathrm{CHB}$ patients with HEV superinfection than those without superinfection, it must be considered that other factors such as patient characteristics, including presence of cirrhosis or other comorbid conditions, and HEV genotype might be different among the studies mentioned, which may have resulted in the contradicting observations noted. Further studies that control for these factors are required to establish the effect of dual infection on viral replication and demonstrate how external factors may contribute to the effects found.

\section{Dual infection in pregnant patients}

The significance of HEV infection in pregnancy is well studied and established. Fulminant hepatic failure, spontaneous abortions and membrane rupture in addition to preterm birth, still birth and neonatal death can occur. ${ }^{42,43}$ However, HEV superinfection in pregnant women with $\mathrm{CHB}$ has not been studied extensively.

One study reported on 200 pregnant patients with chronic liver disease (CLD), $4 \%$ of which had HBV infection (HBsAgpositive). ${ }^{44}$ Of these patients, $1.5 \%$ had positive anti-HEV IgG levels. Non-pregnant patients with CLD $(n=92)$ were also recruited, $32.6 \%$ of which had HBV infection and $18.4 \%$ were positive for anti-HEV IgG, which was a significantly higher rate than the prevalence of HEV in pregnant females (OR:14.7, 95\% CI: 4.1-80.6). Anti-HEV IgM was not detected in any patient in the two groups. AST and ALT levels were significantly higher in anti-HEV IgG-positive patients, when compared to both anti-HEV IgG-negative patients $(p<0.0001)$ and HBV-HCV coinfected patients $(p<0.05)$. The present study showed increased hepatic injury in pregnant patients with $\mathrm{CHB}$ and HEV dual infection. However, this was a single-center study that included a small number of subjects. Additionally, it lacked a control group with HEV infection only.

A larger study recruited 391 pregnant women with positivity for HBsAg and found none of them to be anti-HEV IgMpositive, whereas $42(10.7 \%)$ showed positivity for anti-HEV IgG. ${ }^{45}$ There was no significant difference in anti-HEV IgG positivity between $\mathrm{HBeAg}$-positive or $\mathrm{HBeAg}$-negative patients $(p=0.219)$. When neonatal outcomes (preterm birth rate, gestational age, Apgar score, and birthweight) of patients positive for anti-HEV IgG were compared with antiHEV IgG-negative patients, no significant difference was noted. Transplacental transfer of maternal anti-HEV IgG was noted with a positive correlation between paired maternal and neonatal anti-HEV IgG levels $(r=0.827)$. However, when these neonates were followed for a period of about 10 months, all had undetectable antibody levels at the end of the follow-up period, reflecting natural decay of the maternal antibodies. In postpartum follow up (around 10 months), three women who were initially anti-HEV IgG-negative were found to have detectable levels of the antibody, indicating new infection since the third trimester. However, this was not clinically significant, and these patients remained asymptomatic without derangement in liver function tests. Use of ELISA to detect anti-HEV IgM or IgG may be an important limitation in this study due to its low sensitivity, especially in endemic areas, such as China. Nevertheless, the lack of exacerbation of liver disease in these patients suggests that even if patients had detectable HEV RNA, the infection remained largely subclinical.

It can be inferred from the above study that the incidence of HEV and HBV dual infection in pregnant patients with $\mathrm{CHB}$ is low. However, further studies, especially in other HEV and $\mathrm{HBV}$ endemic parts of the world, are required to establish the frequency of HEV coinfection/dual infection in pregnant patients with $\mathrm{CHB}$ and its clinical implications. The abovementioned study also demonstrated subclinical HEV infection in three patients who showed seroconversion at postpartum follow up.

Ramdasi et al., ${ }^{46}$ in a transcriptome analysis, showed enhanced innate immune response in pregnant patients with self-limiting courses of HEV infection, which may 
explain the subclinical courses of HEV infection in most pregnant patients mentioned in the above studies. However, this study did not include patients with underlying CLD or cirrhosis. Further studies including pregnant patients with underlying CLD who develop HEV superinfection to demonstrate host cell responses are required.

\section{Management}

Acute HEV infection is largely a self-limiting disease that can be managed with supportive care. However, in some severe acute and chronic cases, use of ribavirin (a guanosine analog) has shown some benefit. ${ }^{47-49}$ The goal of treatment is achievement of sustained viral response, which is defined as undetectability of HEV RNA at 12 weeks after stopping treatment. Kumar et al., ${ }^{48}$ in a small prospective study, showed that four out of six kidney transplant patients with acute HEV infection achieved sustained viral response after 3 months of ribavirin therapy. Peron et al. ${ }^{48}$ and Gerolami et al. $^{49}$ demonstrated HEV RNA undetectability within a month after the start of ribavirin therapy. However, since these reports represented case reports and a study with a small population without controls, it is unclear if the viral response was due to ribavirin therapy or to spontaneous resolution. Since immunosuppression is a major risk factor for the development of chronic HEV infection, decreasing the dose of immunosuppression is the first step, as discussed in the following studies.

HBV infection can progress to CLD and is one of the leading causes of cirrhosis and hepatocellular carcinoma. The management strategies of HBV infection have been extensively studied, with established guidelines published, such as by the European Association for the Study of the Liver, Asian Pacific Association for the Study of Liver Disease, and American Association for the Study of Liver Disease. ${ }^{50-52}$ However, there are no established guidelines available on the management of HEV-HBV infections. The use of antivirals, such as sofosbuvir, tenofovir and ribavirin, have been used in certain cases.

Wahid et al. ${ }^{53}$ reported a case of an HBV/HCV/HEV triplepositive patient (with detectable HBV DNA, HCV RNA and HEV RNA). He was treated with tenofovir, daclatasvir, sofosbuvir and ribavirin for 12 weeks, which produced a slow decline in $\mathrm{HBV}$, rapid decline in HCV and complete elimination of the HEV viral load during the first 4 weeks. By week 12 , viral counts for $\mathrm{HBV}$ and HCV were also undetectable and all remained undetectable at 48 weeks post-treatment. The genotype of HEV was not specified in this report.

Conversely, Todesco et al. ${ }^{54}$ reported lack of response to sofosbuvir and ribavirin treatment of HEV, genotype 3 superinfection in a patient with HIV-autoimmune deficiency disease and HBV. For HIV infection, the patient had received tenofovir, emtricitabine, etravirine and raltegravir with HIV and HBV suppression. For the HEV superinfection (HEV RNA-positive), he was initially given ribavirin for 50 months with persistent viremia. He then received intravenous immunoglobulins for 3 months, followed by peginterferon alpha $2 \mathrm{a}$ with ribavirin, but experienced treatment failure again. After this, sofosbuvir with ribavirin was given for 12 weeks, which produced an initial decrease in HEV viral load, followed by a plateau and eventually a steady rise. However, compared to the first case, this patient was relatively severely immunocompromised with CD4 count of $<100 / \mu \mathrm{L}$, which may explain his treatment failure. Large, prospective studies including and comparing patient groups with varying levels of disease severity with healthy controls are required to further assess the effectivity of antiviral treatments for HEV superinfection.

Cheng et al. ${ }^{29}$ found that anti-HBV treatment in patients with $\mathrm{CHB}$ with HEV superinfection did not improve prognosis in terms of liver failure. Of the 294 patients, 118 were assigned to the group of $\mathrm{CHB}$ patients with HEV superinfection and 176 were assigned to the group of acute HEV only. In the former group, only $15(12.7 \%)$ received oral anti-HBV medications, which included lamivudine (3/15) and entecavir (12/ 15). Among the 118 total patients, 42 developed liver failure, out of which $28.6 \%$ received treatment. Out of these 42 patients, mean mortality rates were $66.7 \%$ for those receiving anti-HBV treatment and $53.3 \%$ for those who were not, with no statistical difference found between the two. This retrospective study did not consistently measure HEV RNA. Therefore, contribution of HEV load to disease severity and prognosis could not be ascertained and is a possible confounder.

Chen et al., ${ }^{28}$ in a retrospective study, showed anti-HBV treatment rates to be higher for patients with mild disease $(38.1 \%)$ compared to those with severe disease $(26.0 \%$, $p=0.281)$ and higher for patients who survived $(52.8 \%)$ compared to those who did not $(30 \%, p=0.081)$. However, these differences were not statistically significant and anti-HBV treatment was not found to be significantly associated with clinical outcomes. Prospective studies might be more useful in determining the effect of anti-HBV treatment on the outcomes of patients with HEV superinfection.

\section{Vaccines}

In a study of prevention, a double-blind, randomized, placebo-controlled, phase 3 trial regarding efficacy and safety of recombinant hepatitis $\mathrm{E}$ vaccine in healthy adults was conducted in China. ${ }^{55}$ In this, 56,302 healthy individuals were assigned to the vaccine group and 56,302 healthy individuals were assigned to the placebo group. From each group, $86 \%$ received three doses of the HEV 239 (HEV vaccine). The study population was followed for 12 months after 30 days from receiving the third dose of vaccine. In the placebo group, 15 participants developed HEV infection (anti-HEV IgM- or IgG-positive and/or HEV RNA-positive), whereas none developed infection in the vaccine group. Thus, the vaccine efficacy was $100.0 \%$ after three doses (95\% CI: 72.1-100.0). This large clinical trial, with a favorable study design (randomized, double-blinded), demonstrated the anti-HEV vaccine to be potentially preventative, which may help to decrease the incidence of HEV infection. However, this vaccine should be prioritized in patients with chronic liver diseases, such as $\mathrm{CHB}$, but this study excluded patients with CLD, which is a major limitation. Additionally, the vaccine is only licensed in China and is not available in other parts of the world.

\section{Conclusions}

HEV superinfection in patients with underlying $\mathrm{CHB}$ can result in an acute exacerbation of their liver disease. In patients with cirrhosis, a superinfection can result in decompensated liver disease and increase mortality. Immunosuppression is a risk factor for development of chronic HEV disease. There are no established guidelines on the management of HEV-HBV dual infection. To this end, human-induced pluripotent stem cells 
to generate functional hepatic organoids could be used to model HBV-HEV dual infection and in studies to make observations regarding the virus-host interactions and the effects of viruses on each other's replication and response to therapies. We suggest a multifaceted approach for the management of HEV-HBV dual infection. For the treatment of HEV, the dose of immunosuppressant should be decreased if applicable, and ribavirin should be given for at least 12 weeks. Following this, HEV RNA in serum or stool should be checked. If there is detectable HEV RNA, ribavirin should be continued for an additional 12 weeks. This treatment should be accompanied by management of HBV with nucleoside/ nucleotide analogs, such as tenofovir or entecavir. However, it is important to note that HBV is a vaccine-preventable disease and focus should be placed on timely vaccination and prevention of HBV. An HEV vaccine is approved and used in practice in China but has not been tested in CHB patients. Worldwide approval and availability of this vaccine can be a cornerstone in prevention of this disease. HEV vaccine administration should be prioritized for patients with underlying liver disorders and immunocompromised patients. Lastly, patient education, especially in endemic areas, regarding the transmission of HBV and HEV will be imperative in controlling and preventing the spread of the viruses.

\section{Acknowledgments}

Support of the Herman Lopata Chair in Hepatitis is gratefully acknowledged.

\section{Funding}

None to declare.

\section{Conflict of interest}

The authors have no conflict of interests related to this publication.

\section{Author contributions}

Wrote and revised the review article (MN), edited the review article (GYW).

\section{References}

[1] World Health Organization. Hepatitis E. Available from: https://www.who. int/en/news-room/fact-sheets/detail/hepatitis-e.

[2] Boccia D, Guthmann JP, Klovstad H, Hamid N, Tatay M, Ciglenecki I, et al. High mortality associated with an outbreak of hepatitis $\mathrm{E}$ among displaced persons in Darfur, Sudan. Clin Infect Dis 2006;42:1679-1684. doi: 10. $1086 / 504322$

[3] Dalton HR, Bendall RP, Keane FE, Tedder RS, Ijaz S. Persistent carriage of hepatitis E virus in patients with HIV infection. N Engl J Med 2009;361:10251027. doi: 10.1056/NEJMc0903778.

[4] Kamar N, Mansuy JM, Cointault O, Selves J, Abravanel F, Danjoux M, et al. Hepatitis E virus-related cirrhosis in kidney- and kidney-pancreas-transplant recipients. Am J Transplant 2008;8:1744-1748. doi: 10.1111/j.1600-6143. 2008.02286.x.

[5] Arankalle VA, Chobe LP. Hepatitis E virus: can it be transmitted parenterally? J Viral Hepat 1999;6:161-164. doi: 10.1046/j.1365-2893.1999.00141.x.

[6] Khuroo MS, Kamili S, Yattoo GN. Hepatitis E virus infection may be transmitted through blood transfusions in an endemic area. J Gastroenterol Hepatol 2004;19:778-784. doi: 10.1111/j.1440-1746.2004.03437.x.

[7] Kumar M, Sharma BC, Sarin SK. Hepatitis E virus as an etiology of acute exacerbation of previously unrecognized asymptomatic patients with hepa- titis B virus-related chronic liver disease. J Gastroenterol Hepatol 2008;23: 883-887. doi: 10.1111/j.1440-1746.2007.05243.x.

[8] Jagya N, Varma SP, Thakral D, Joshi P, Durgapal H, Panda SK. RNA-seq based transcriptome analysis of hepatitis E virus (HEV) and hepatitis B virus (HBV) replicon transfected Huh-7 cells. PLoS One 2014;9:e87835. doi: 10. 1371/journal.pone.0087835.

[9] Dickson RC, Everhart JE, Lake JR, Wei Y, Seaberg EC, Wiesner RH, et al. Transmission of hepatitis $B$ by transplantation of livers from donors positive for antibody to hepatitis B core antigen. The National Institute of Diabetes and Digestive and Kidney Diseases Liver Transplantation Database. Gastroenterology 1997;113:1668-1674. doi: 10.1053/gast.1997.v113. pm9352871.

[10] Price J. An update on hepatitis B, D, and E viruses. Top Antivir Med 2014;21: 157-163.

[11] Xiong LS, Cui SF, Zhou JG, Xing Y. Detection and analysis of HAV-HEV, HGV infection in patients with viral hepatitis. Zhonghua Gan Zang Bing Za Zhi 2004;12:395-396.

[12] Bayram A, Eksi F, Mehli M, Sözen E. Prevalence of hepatitis E virus antibodies in patients with chronic hepatitis B and chronic hepatitis C. Intervirology 2007;50:281-286. doi: 10.1159/000103916.

[13] Singh NJ, Kumari A, Catanzaro R, Marotta F. Prevalence of hepatitis E and hepatitis B dual infection in North India (Delhi). Acta Biomed 2012;83:197201.

[14] Zhou X, de Man RA, de Knegt RJ, Metselaar HJ, Peppelenbosch MP, Pan Q. Epidemiology and management of chronic hepatitis $E$ infection in solid organ transplantation: a comprehensive literature review. Rev Med Virol 2013;23: 295-304. doi: 10.1002/rmv.1751.

[15] Kamar N, Garrouste C, Haagsma EB, Garrigue V, Pischke S, Chauvet C, et al. Factors associated with chronic hepatitis in patients with hepatitis $E$ virus infection who have received solid organ transplants. Gastroenterology 2011;140:1481-1489. doi: 10.1053/j.gastro.2011.02.050.

[16] Piroth L, Sène D, Pol S, Goderel I, Lacombe K, Martha B, et al. Epidemiology, diagnosis and treatment of chronic hepatitis B in HIV-infected patients (EPIB 2005 STUDY). AIDS 2007;21:1323-1331. doi: 10.1097/QAD. Ob013e32810c8bcf.

[17] Sherman KE, Terrault N, Barin B, Rouster SD, Shata MT. Hepatitis E infection in HIV-infected liver and kidney transplant candidates. J Viral Hepat 2014; 21:e74-e77. doi: 10.1111/jvh.12233.

[18] Rapicetta M, Monarca R, Kondili LA, Chionne P, Madonna E, Madeddu G, et al. Hepatitis $E$ virus and hepatitis $A$ virus exposures in an apparently healthy high-risk population in Italy. Infection 2013;41:69-76. doi: 10 . 1007/s15010-012-0385-8.

[19] Hunt DR, Saab S. Viral hepatitis in incarcerated adults: a medical and public health concern. Am J Gastroenterol 2009;104:1024-1031. doi: 10. 1038/ajg.2008.143.

[20] Mobaien AR, Mohammadi R, Sorouri R, Sadeghi K. Hepatitis E virus seroprevalence in haemodialysis patients in Zanjan Province, Islamic Republic of Iran. East Mediterr Health J 2013;19:608-612. doi: 10.26719/2013.19.7. 608.

[21] Kumar Acharya S, Kumar Sharma P, Singh R, Kumar Mohanty S, Madan K, Kumar Jha J, et al. Hepatitis E virus (HEV) infection in patients with cirrhosis is associated with rapid decompensation and death. J Hepatol 2007;46:387394. doi: 10.1016/j.jhep.2006.09.016.

[22] Hamid SS, Atiq M, Shehzad F, Yasmeen A, Nissa T, Salam A, et al. Hepatitis E virus superinfection in patients with chronic liver disease. Hepatology 2002; 36:474-478. doi: 10.1053/jhep.2002.34856.

[23] Kumar A, Aggarwal R, Naik SR, Saraswat V, Ghoshal UC, Naik S. Hepatitis E virus is responsible for decompensation of chronic liver disease in an endemic region. Indian J Gastroenterol 2004;23:59-62.

[24] Zhao J, Shi J, Qu M, Zhao X, Wang H, Huang M, et al. Hyperactive follicular helper $T$ cells contribute to dysregulated humoral immunity in patients with liver cirrhosis. Front Immunol 2019;10:1915. doi: 10.3389/fimmu.2019. 01915.

[25] Albillos A, Lario M, Álvarez-Mon M. Cirrhosis-associated immune dysfunction: distinctive features and clinical relevance. J Hepatol 2014;61:13851396. doi: 10.1016/j.jhep.2014.08.010.

[26] Lefilliatre $P$, Villeneuve JP. Fulminant hepatitis $A$ in patients with chronic liver disease. Can J Public Health 2000;91:168-170. doi: 10.1007/BF03404264.

[27] Fattovich G, Giustina G, Christensen E, Pantalena M, Zagni I, Realdi G, et al. Influence of hepatitis delta virus infection on morbidity and mortality in compensated cirrhosis type B. The European Concerted Action on Viral Hepatitis (Eurohep). Gut 2000;46:420-426. doi: 10.1136/gut.46.3.420.

[28] Chen C, Zhang SY, Zhang DD, Li XY, Zhang YL, Li WX, et al. Clinical features of acute hepatitis $E$ super-infections on chronic hepatitis B. World J Gastroenterol 2016;22:10388-10397. doi: 10.3748/wjg.v22.i47.10388.

[29] Cheng SH, Mai L, Zhu FQ, Pan XF, Sun HX, Cao H, et al. Influence of chronic HBV infection on superimposed acute hepatitis E. World J Gastroenterol 2013;19:5904-5909. doi: 10.3748/wjg.v19.i35.5904.

[30] Chow CW, Tsang SW, Tsang OT, Leung VK, Fung KS, Luk WK, et al. Comparison of acute hepatitis $E$ infection outcome in patients with and without 
chronic hepatitis $B$ infection: a 10 year retrospective study in three regional hospitals in Hong Kong. J Clin Virol 2014;60:4-10. doi: 10.1016/j.jcv.2014. 01.024 .

[31] Kilonzo SB, Wang $Y L$, Jiang QQ, Wu WY, Wang $P$, Ning $Q$, et al. Superinfective hepatitis $E$ virus infection aggravates hepatocytes injury in chronic hepatitis B. Curr Med Sci 2019;39:719-726. doi: 10.1007/s11596-019-2097-0.

[32] Fu J, Guo D, Gao D, Huang W, Li Z, Jia B. Clinical analysis of patients suffering from chronic hepatitis B superinfected with other hepadnaviruses. J Med Virol 2016;88:1003-1009. doi: 10.1002/jmv.24417.

[33] Lai JC, Wong GL, Yip TC, Tse YK, Lam KL, Lui GC, et al. Chronic hepatitis B increases liver-related mortality of patients with acute hepatitis $\mathrm{E}$ : A territorywide cohort study from 2000 to 2016. Clin Infect Dis 2018;67:12781284. doi: $10.1093 /$ cid/ciy234.

[34] Li Q, Chen C, Huang C, Xu W, Hu Q, Chen L. Noninvasive models for predicting poor prognosis of chronic HBV infection patients precipitating acute HEV infection. Sci Rep 2020;10:2753. doi: 10.1038/s41598-020-59670-4.

[35] Bouamra Y, Benali S, Tissot-Dupont H, Tamalet C, Colson P. Hepatitis B and E co-primary infections in an HIV-1-infected patient. J Clin Microbiol 2013;51: 1052-1056. doi: 10.1128/JCM.02630-12.

[36] Barde PV, Chouksey VK, Shivlata L, Sahare LK, Thakur AK. Viral hepatitis among acute hepatitis patients attending tertiary care hospital in central India. Virusdisease 2019;30:367-372. doi: 10.1007/s13337-019-00541-6.

[37] Zhang X, Ke W, Xie J, Zhao Z, Xie D, Gao Z. Comparison of effects of hepatitis $E$ or $A$ viral superinfection in patients with chronic hepatitis B. Hepatol Int 2010;4:615-620. doi: 10.1007/s12072-010-9204-4.

[38] Yeh CT, Yeh CS, Chu YD, Chiang Y]. Seroclearance of hepatitis B surface antigen following hepatitis $\mathrm{E}$ exacerbation on chronic hepatitis $\mathrm{E}$ and $\mathrm{B}$ dual infection in a renal transplant recipient: a case report. J Med Case Rep 2018; 12:50. doi: 10.1186/s13256-018-1586-2.

[39] Hoan NX, Tong HV, Hecht N, Sy BT, Marcinek P, Meyer CG, et al. Hepatitis E virus superinfection and clinical progression in hepatitis $B$ patients. EBioMedicine 2015;2:2080-2086. doi: 10.1016/j.ebiom.2015.11.020.

[40] Schulz M, Schott E. An unusual cause for a hepatic flare in a chronic HBV carrier. Hepat Mon 2014;14:e20099. doi: 10.5812/hepatmon.20099.

[41] Shang Q, Yu J, Xiao D, Xu C, Chen C, Zhang G. The effects of hepatitis E virus superinfection on patients with chronic hepatitis B: a clinico-pathological study. Zhonghua Nei Ke Za Zhi 2002;41:656-659.

[42] Patra S, Kumar A, Trivedi SS, Puri M, Sarin SK. Maternal and fetal outcomes in pregnant women with acute hepatitis $E$ virus infection. Ann Intern Med 2007;147:28-33. doi: 10.7326/0003-4819-147-1-200707030-00005.

[43] Shinde N, Patil T, Deshpande A, Gulhane R, Patil M, Bansod Y. Clinical profile, maternal and fetal outcomes of acute hepatitis e in pregnancy. Ann Med Health Sci Res 2014;4:S133-S139. doi: 10.4103/2141-9248.138033.
[44] Sévédé $D$, Doumbia $M$, Kouakou $V$, Djehiffe $V$, Pineau $P$, Dosso $M$. Increased liver injury in patients with chronic hepatitis and IgG directed against hepatitis E virus. EXCLI J 2019;18:955-961. doi: 10.17179/excli2019-1827.

[45] Huang $H$, Xu C, Zhou X, Liu L, Dai $Y, X u B$, et al. Incidence and seroprevalence of hepatitis $E$ virus infection in pregnant women infected with hepatitis $B$ virus and antibody placental transfer in infants. J Clin Virol 2016;82:8488. doi: 10.1016/j.jcv.2016.07.010.

[46] Ramdasi AY, Arankalle VA. The expression patterns of immune response genes in the Peripheral Blood Mononuclear cells of pregnant women presenting with subclinical or clinical HEV infection are different and trimesterdependent: A whole transcriptome analysis. PLoS One 2020;15:e0228068. doi: 10.1371 /journal.pone.0228068.

[47] Kamar N, Rostaing L, Abravanel F, Garrouste C, Lhomme S, Esposito L, et al. Ribavirin therapy inhibits viral replication on patients with chronic hepatitis e virus infection. Gastroenterology 2010;139:1612-1618. doi: 10.1053/j. gastro.2010.08.002.

[48] Péron JM, Dalton H, Izopet J, Kamar N. Acute autochthonous hepatitis E in western patients with underlying chronic liver disease: a role for ribavirin? J Hepatol 2011;54:1323-1324; author reply 1324-1325. doi: 10.1016/j.jhep. 2011.01.009.

[49] Gerolami R, Borentain P, Raissouni F, Motte A, Solas C, Colson P. Treatment of severe acute hepatitis E by ribavirin. J Clin Virol 2011;52:60-62. doi: 10. 1016/j.jcv.2011.06.004.

[50] Sarin SK, Kumar M, Lau GK, Abbas Z, Chan HL, Chen C], et al. Asian-Pacific clinical practice guidelines on the management of hepatitis B: a 2015 update. Hepatol Int 2016;10:1-98. doi: 10.1007/s12072-015-9675-4.

[51] EASL 2017 Clinical Practice Guidelines on the management of hepatitis B virus infection. J Hepatol 2017;67:370-398. doi: 10.1016/j.jhep.2017.03. 021.

[52] Terrault NA, Lok ASF, McMahon BJ, Chang KM, Hwang JP, Jonas MM, et al. Update on prevention, diagnosis, and treatment of chronic hepatitis $B$ : AASLD 2018 hepatitis B guidance. Hepatology 2018;67:1560-1599. doi: 10.1002/hep. 29800 .

[53] Wahid B. Successful treatment of HBV, HCV, \& HEV, with 12-week long use of tenofovir, sofosbuvir, daclatasvir, and ribavirin: A case report. J Infect Public Health 2020;13:149-150. doi: 10.1016/j.jiph.2019.06.004.

[54] Todesco E, Demeret S, Calin R, Roque-Afonso AM, Thibault V, Mallet V, et al. Chronic hepatitis $E$ in HIV/HBV coinfected patient: lack of power of sofosbuvir-ribavirin. AIDS 2017;31:1346-1348. doi: 10.1097/QAD. 0000000000001474.

[55] Zhu FC, Zhang J, Zhang XF, Zhou C, Wang ZZ, Huang SJ, et al. Efficacy and safety of a recombinant hepatitis $E$ vaccine in healthy adults: a large-scale, randomised, double-blind placebo-controlled, phase 3 trial. Lancet 2010; 376:895-902. doi: 10.1016/S0140-6736(10)61030-6. 